\title{
Obvious optic disc swelling in a patient with cryopyrin-associated periodic syndrome
}

This article was published in the following Dove Press journal:

Clinical Ophthalmology

5 August 2013

Number of times this article has been viewed

\author{
Mariko Kawai' \\ Tadanobu Yoshikawa' \\ Ryuta Nishikomori ${ }^{2}$ \\ Toshio Heike ${ }^{2}$ \\ Kanji Takahashi' \\ 'Department of Ophthalmology, \\ Kansai Medical University, Osaka, \\ ${ }^{2}$ Department of Pediatrics, Graduate \\ School of Medicine, Kyoto University, \\ Kyoto, Japan
}

\begin{abstract}
Cryopyrin-associated periodic syndrome (CAPS) is a group of rare hereditary autoinflammatory diseases caused by mutations of the NLRP3 gene, and leads to excessive production of the proinflammatory cytokine, interleukin-1 $\beta$. A 35-year-old male presented with recurrent symptoms of urticarial-like rash, periodic fever, arthralgia, headache, and eye redness. His best-corrected visual acuity was 1.0 OD and 0.9 OS. Slit-lamp examination showed conjunctival and episcleral injection in both eyes. Ophthalmoscopy revealed obvious bilateral optic disc swelling and retinal vascular sheathing around the optic discs. Spectral domain optical coherence tomography also showed obvious optic disc swelling. Steroid and nonsteroidal antiinflammatory drugs did not improve these symptoms. Genetic testing detected a heterozygous mutation of c. $907 \mathrm{G}>$ A. Thus, the patient was genetically confirmed with CAPS. Visual acuity did not decrease for 3 years, although the optic discs became white in color. CAPS should therefore be distinguished from other disorders when examining optic disc swelling and/or uveitis patients with urticarial-like rash and periodic fever.
\end{abstract}

Keywords: interleukin-1 $\beta$, chronic infantile cutaneous and articular syndrome, cryopyrinassociated periodic syndrome, leucine-rich repeat-containing protein 3, optic disc swelling

\section{Introduction}

Cryopyrin-associated periodic syndrome (CAPS) is a group of rare hereditary autoinflammatory diseases, and includes the familial cold autoinflammatory syndrome, Muckle-Wells syndrome, chronic infantile cutaneous and articular syndrome (CINCA), and neonatal-onset multisystem inflammatory disease (NOMID). ${ }^{1}$ Autoinflammatory syndromes are systemic disorders which result in repeated inflammatory symptoms such as fever, rash, and arthritis in the absence of autoantibodies with high titer or antigen-specific T lymphocytes. ${ }^{1}$ It is different from infection, autoimmunity, allergy, and immunodeficiency. ${ }^{1}$ CAPS is caused by gain-of-function mutations in the nucleotide-binding domain and leucine-rich repeat-containing protein 3 (NLRP3) within the cryopyrin gene. ${ }^{2}$ NLRP3 mutations increase activity of the interleukin-1 (IL-1) inflammasome and lead to excessive production of IL-1 $\beta$, a proinflammatory cytokine. ${ }^{2}$ Thus, genetic testing is necessary for diagnosis of CAPS. ${ }^{2}$ CAPS is an autosomal dominant disease and the syndrome generally presents in early childhood. ${ }^{3}$ Clinical findings include urticarial-like rash, periodic fever, inflammation of the central nervous system, meningitis, optic disc swelling, deafness, and arthropathy. ${ }^{3-5}$ Ocular manifestations of CAPS are not fully understood, although it is known to cause optic disc changes, anterior uveitis, and corneal involvement. ${ }^{4}$ In this case study, we report CAPS associated with optic disc swelling.
Correspondence: Tadanobu Yoshikawa Kansai Medical University, Department of Ophthalmology, 2-5-I Shinmachi, Hirakata, Osaka, Japan

Tel +810728040101

Fax +8I 0728042039

Email yoshikat@takii.kmu.ac.jp 


\section{Case report}

In 2009, a 35-year-old Japanese male visited the dermatology clinic of our hospital presenting with urticarial-like rash from infancy, periodic fever, and arthralgia. He also presented with deafness, repeated eye redness, ocular pain, headache, and general fatigue from the time he was a child. He had a previous history of meningitis at the age of 20 years. At first, Behçet's disease was suspected based on his symptoms. Consequently, he was seen for diagnosis in the ophthalmology clinic at our hospital in September 2009. His best-corrected visual acuity was 1.0 OD and 0.9 OS and his manifest refraction was uncorrected OU. Intraocular pressure was $9 \mathrm{mmHg}$ OD and $7 \mathrm{mmHg}$ OS. Slit-lamp examination showed conjunctival and episcleral injection in both eyes, clear and smooth corneas, and infiltration of inflammatory cells without posterior synechia in the anterior chamber. Gonioscopy did not reveal hypopyon, nodules, or peripheral anterior synechia. Ophthalmoscopy and fundus photography examination revealed obvious bilateral optic disc swelling and retinal vascular sheathing around the optic discs, with absence of dilated capillaries on the optic discs, vitreous opacifications, and retinal exudates (Figure 1). Critical flicker frequency was normal $(43.8 \mathrm{~Hz}$ OD and $47.2 \mathrm{~Hz}$ OS). Goldmann perimetry showed only enlarged blind spots in both eyes. The patient had no ocular findings of Behçet's disease. Hematologic examination showed increased inflammatory markers, a white blood cell count of 18,700 cells/ $\mu \mathrm{L}$ (neutrophil count $75.5 \%$, lymphocyte count $20.5 \%$ ), C-reactive protein of $2.94 \mathrm{mg} / \mathrm{dL}$, and an erythrocyte sedimentation rate of $44 \mathrm{~mm}$. Magnetic resonance imaging showed no intracranial space-occupying lesion.
The anterior uveitis was treated with steroid and nonsteroidal anti-inflammatory eye drops. The patient was also treated at the department of rheumatology and clinical immunology with immunosuppressive agents (methotrexate, tacrolimus, azathioprine, cyclophosphamide) and antirheumatic agents (salazosulfapyridine, leflunomide) to reverse systemic symptoms. However, conjunctival and episcleral injection and systemic symptoms played a limited role in these treatments. Three years later, his best-corrected visual acuity was $1.2 \mathrm{OD}$ and $1.2 \mathrm{OS}$. His conjunctival and episcleral injection remained unchanged (Figure 2). The optic discs became white in color (Figure 1) and fluorescein angiography demonstrated strong staining and weak late phase leakage from the optic disc (Figure 3). Spectral domain optical coherence tomography (Spectralis, Heidelberg Engineering, Heidelberg, Germany) revealed obvious optic disc swelling (Figure 1).

In 2012, his mother was genetically diagnosed with CAPS. Therefore, the patient underwent genetic testing for the diagnosis of CAPS at Kyoto University Graduate School of Medicine after informed consent was obtained, according to the tenets of the Declaration of Helsinki. Genetic tests detected heterozygous mutations in c. $907 \mathrm{G}>\mathrm{A}$ [p.Asp303Asn $(\mathrm{D} 303 \mathrm{~N})]$, a previously reported mutation responsible for CAPS. Thus, he received a definitive diagnosis of CAPS.

\section{Discussion}

CAPS consists of three clinical categories, and the familial cold autoinflammatory syndrome is the mildest condition. ${ }^{3}$ The Muckle-Wells syndrome presents an intermediate con-
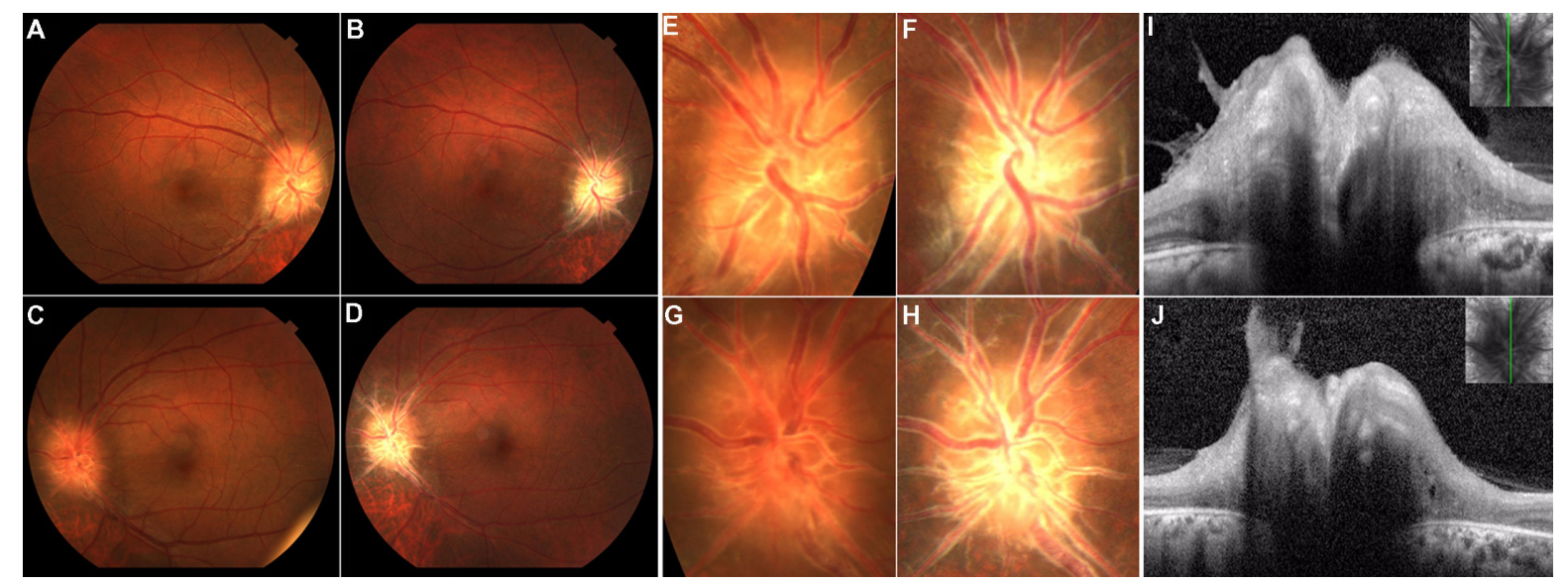

Figure I Fundus photographs of the right eye ( $\mathbf{A}$ and $\mathbf{B})$ and left eye (C and $\mathbf{D})$, and photographs focused on the optic disc of the right eye (E and $\mathbf{F})$ and left eye ( $\mathbf{G}$ and $\mathbf{H})$ taken in 2009 and 2012, respectively. Spectral-domain optical coherence tomography scans of the optic disc in the right eye (I) and left eye (J) in 20I2. (A-D) Fundus photographs show obvious bilateral optic disc swelling, and absence of vitreous opacifications and retinal exudates. (E and $\mathbf{F}$ ) Fundus photographs focused on the optic disc reveal the lack of a sharp outline to the redness of the optic discs, and retinal vascular sheathing around the optic discs. There are no dilated superficial capillaries and hemorrhages on optic discs as was observed in 2009. ( $\mathbf{G}$ and $\mathbf{H}$ ) Both optic discs were white in color and retinal vascular sheathing was increased after 3 years. (I and J) Longitudinal scans of the optic discs showing obvious optic disc swelling. 


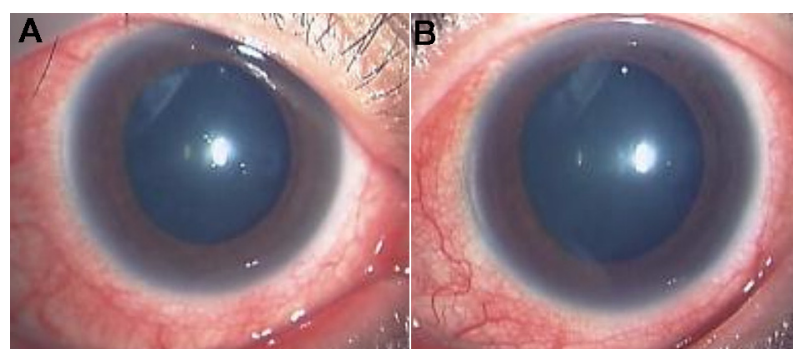

Figure 2 Slit-lamp examinations of the right eye (A) and left eye (B) in 2012. Slit-lamp biomicroscopy revealed strong conjunctivitis and episcleritis in both eyes treated with mydriatics.

dition between familial cold autoinflammatory syndrome and CINCA/NOMID. ${ }^{3}$ CINCA/NOMID is the most severe condition. ${ }^{3}$ Common manifestations include urticarial-like rash and periodic fever. ${ }^{3}$ Clinical criteria for diagnosis of each individual syndrome have been previously reported. ${ }^{6-8}$ According to these reports, CINCA/NOMID presents with urticarial-like rash, periodic fever, inflammation of the central nervous system, and arthropathy. ${ }^{6-8}$ Our patient presented with a severe phenotype, including skin erythema, arthralgia, inflammation of the central nervous system, optic disc swelling, meningitis, and deafness. Additionally, alteration of the nucleotide c. $907 \mathrm{G}>\mathrm{A}$ was detected, and previously has been reported as being associated with the phenotypes of CINCA/NOMID and Muckle-Wells syndromes. ${ }^{9}$ Consequently, we identified this patient as having CINCA/ NOMID or Muckle-Wells syndrome.

The International CINCA/NOMID Ocular Study Group reported ocular manifestations of CINCA/NOMID in $2000 .{ }^{4}$ However, at that time CAPS had no known cause. According to the report, of 31 patients, 26 (84\%) had optic disc changes, including optic disc edema, pseudopapilledema, and optic atrophy, 13 patients (42\%) presented with chronic eye redness, including chronic perilimbal injection and chronic conjunctivitis, 17 patients (55\%) had chronic anterior uveitis, and none of the patients had increased intraocular pressure or glaucoma. ${ }^{4}$ Our case also demonstrated optic disc changes, chronic conjunctivitis, episcleritis, and anterior uveitis. We think that the optic disc change in our case is optic edema, because fluorescein angiography demonstrated late phase leakage and ophthalmoscopy showed retinal vascular sheathing.

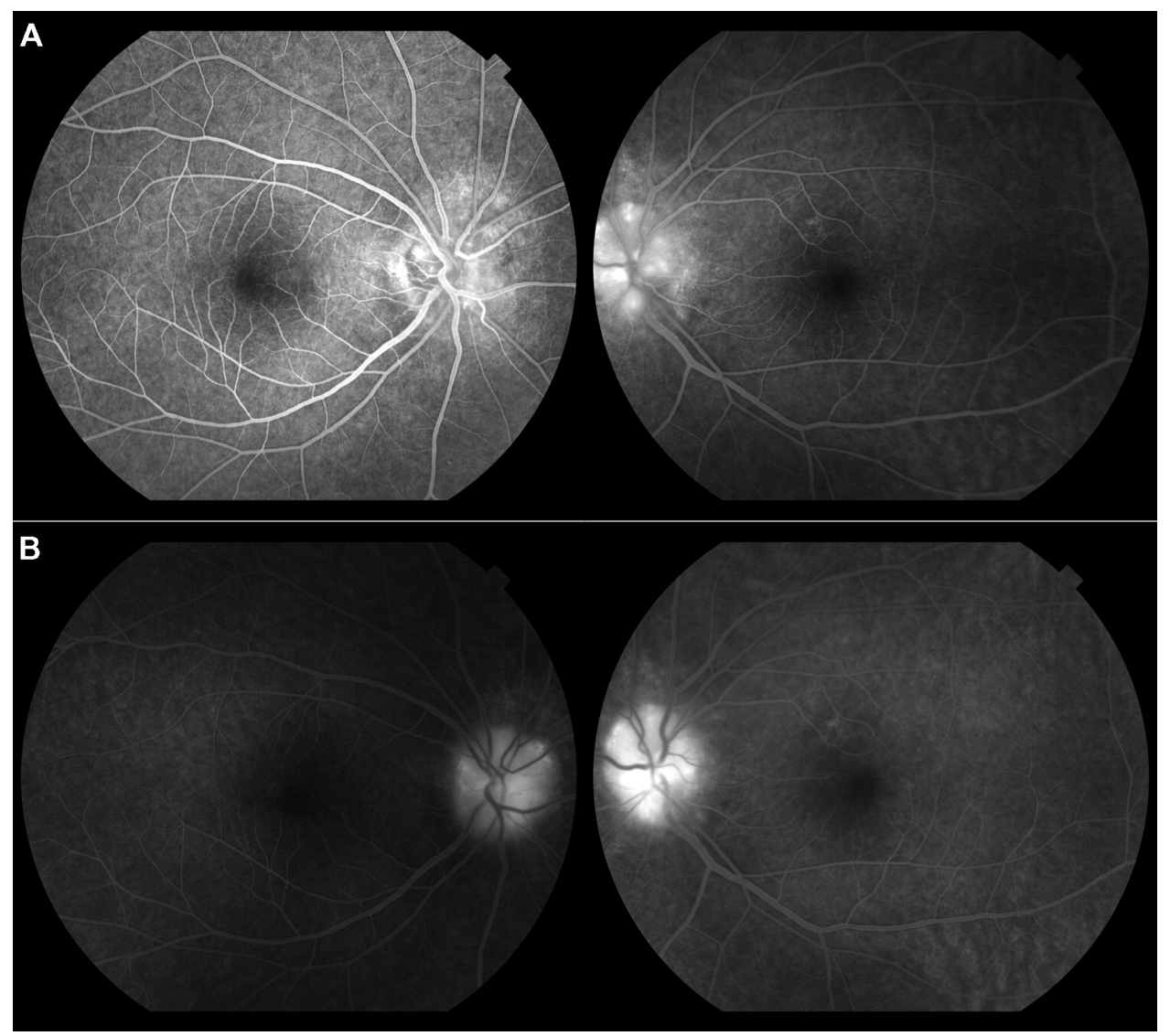

Figure 3 Fluorescein angiography at early phase (A) and late phase (B) in 2012. Fluorescein angiography showed strong staining and weak late phase leakage from the optic disc in both eyes. There were no dilated superficial capillaries on optic discs. 
Terrada et al reported a case of CAPS, and the patient had optic disc changes related to chronic intracranial hypertension due to cerebrospinal fluid inflammation. ${ }^{10}$ We do not know whether our patient presented with intracranial hypertension because lumbar puncture was not performed in this case. However, there were no space-occupying brain lesions produced by raised intracranial pressure observed with magnetic resonance imaging.

We speculate two possibilities for optic disc change in our case. First, the optic disc change in our patient may be caused by ocular specific inflammation, because ophthalmoscopy revealed retinal vascular sheathing around the optic discs. Second, inflammation of the central nervous system may lead to optic disc change, because he had a previous history of meningitis. We hypothesize that the optic disc change in CAPS was due to ocular specific inflammation, inflammation of the central nervous system, or raised intracranial pressure.

The CINCA/NOMID Group reported that 19 of 30 patients with CINCA/NOMID had adequate visual acuity or mild visual loss. ${ }^{4}$ The patient in our study also had adequate visual acuity. CINCA/NOMID generally occurs with severe systemic symptoms and optic disc changes. ${ }^{4}$ However, the report found only eight patients (27\%) had severe visual loss. ${ }^{4}$ The authors hypothesized that a cause may be the lack of long-term systematic review in many patients. ${ }^{4}$ The duration of clinical follow-up in our case was also approximately 4 years. In the future, our patient may have severe visual acuity loss.

Usually steroids, nonsteroidal anti-inflammatory drugs, and immunosuppressive agents are not effective for CAPS. ${ }^{3}$ In our study, these drugs had little effect on the patient's symptoms. CAPS is caused by mutations of the NLRP3 gene that encodes for cryopyrin protein. ${ }^{2}$ Cryopyrin protein is part of an inflammasome complex, and regulates production and secretion of the potent proinflammatory cytokine IL- $1 \beta$ in blood myeloid cells. ${ }^{2}$ Thus, anti-inflammatory drugs directed at IL-1, such as anakinra, rilonacept, and canakinumab, are used for treatment of CAPS. ${ }^{11-13}$

In conclusion, we showed that the main ocular findings in our case were optic disc swelling, chronic conjunctivitis, episcleritis, and chronic anterior uveitis, without elevated intraocular pressure and peripheral anterior synechia. Steroid and nonsteroidal anti-inflammatory drugs did not improve these symptoms. CAPS is a rare ophthalmologic syndrome and little is known of its cause. Hence, clinicians must distinguish CAPS from other disorders when examining optic disc swelling and/or uveitis patients with urticarial-like rash and periodic fever.

\section{Author contributions}

The authors alone are responsible for the content and writing of the paper and agree to allow Clinical Ophthalmology to review their data upon request.

\section{Disclosure}

The authors report no conflicts of interest.

\section{References}

1. Masters SL, Simon A, Aksentijevich I, Kastner DL. Horror autoinflammaticus: the molecular pathophysiology of autoinflammatory disease $(*)$. Annu Rev Immunol. 2009;27:621-668.

2. Hoffman HM, Wanderer AA. Inflammasome and IL-1 $\beta$-mediated disorders. Curr Allergy Asthma Rep. 2010;10:229-235.

3. Yu JR, Leslie KS. Cryopyrin-associated periodic syndrome: an update on diagnosis and treatment response. Curr Allergy Asthma Rep. 2011;11:12-20.

4. Chronic Infantile Neurological Cutaneous and Articular/Neonatal Onset Multisystem Inflammatory Disease (CINCA/NOMID) Ocular Study Group. Chronic infantile neurological cutaneous and articular/neonatal onset multisystem inflammatory disease syndrome. Arch Ophthalmol. 2000;118:1386-1392.

5. Goldbach RM. Current status of understanding the pathogenesis and management of patients with NOMID/CINCA. Curr Rheumatol Rep. 2011;13:123-131

6. Hoffman HM, Wanderer AA, Broide DH. Familial cold autoinflammatory syndrome: phenotype and genotype of an autosomal dominant periodic fever. J Allergy Clin Immunol. 2001;108:615-620.

7. Goldbach RM, Dailey NJ, Canna SW, et al. Neonatal onset multisystem inflammatory disease responsive to interleukin-1 beta inhibition. $N E n g l$ J Med. 2006;355:581-592.

8. Neven B, Callebaut I, Prieur AM, et al. Molecular basis of the spectral expression of CIAS1 mutations associated with phagocytic cellmediated autoinflammatory disorders CINCA/NOMID, MWS, and FCU. Blood. 2004;103:2809-2815.

9. Dodé C, Le Dû N, Cuisset L, et al. New mutations of CIAS1 that are responsible for Muckle-Wells syndrome and familial cold urticaria: a novel mutation underlies both syndromes. Am J Hum Genet. 2002;70:1498-1506.

10. Terrada C, Neven B, Boddaert N, et al. Ocular modifications in a young girl with cryopyrin-associated periodic syndromes responding to interleukin-1 receptor antagonist anakinra. J Ophthalmic Inflamm Infect. 2011;1:133-136.

11. Hoffman HM, Rosengren S, Boyle DL, et al. Prevention of cold-associated acute inflammatory in familial cold autoinflammatory syndrome by interleukin-1 receptor antagonist. Lancet. 2004;364:1779-1785.

12. Hoffman HM, Throne ML, Amar NJ, et al. Efficacy and safety of rilonacept(interleukin-1 trap) in patients with cryopyrin-associated periodic syndromes. Results from two sequential placebo-controlled studies. Arthritis Rheum. 2008;58:2443-2452.

13. The Canakinumab in CAPS Study Group. Use of canakinumab in the cryopyrin-associated periodic syndrome. N Engl J Med. 2009;360: 2416-2425. 
Clinical Ophthalmology

\section{Publish your work in this journal}

Clinical Ophthalmology is an international, peer-reviewed journal covering all subspecialties within ophthalmology. Key topics include: Optometry; Visual science; Pharmacology and drug therapy in eye diseases; Basic Sciences; Primary and Secondary eye care; Patien Safety and Quality of Care Improvements. This journal is indexed on

PubMed Central and CAS, and is the official journal of The Society of Clinical Ophthalmology (SCO). The manuscript management system is completely online and includes a very quick and fair peer-review system, which is all easy to use. Visit http://www.dovepress.com/ testimonials.php to read real quotes from published authors. 